\title{
THE BISPECTRAL ALIASING TEST: A CLARIFICATION AND SOME KEY EXAMPLES
}

\author{
Kevin R. Vixie, Murray Wolinsky and David E. Sigeti \\ Los Alamos National Laboratory \\ Los Alamos, NM 87545 \\ murray@lanl.gov
}

\begin{abstract}
Controversy regarding the correctness of a test for aliasing proposed by Hinich and Wolinsky [3] has been surprisingly long-lived. Two factors have prolonged this controversy. One factor is the presence of deep-seated intuitions that such a test is fundamentally incoherent. Perhaps the most compelling objection is that, given a set of discretetime samples, one can construct an unaliased continuoustime series which exactly fits those samples. Therefore, the samples alone can not show that the original time series was aliased. The second factor prolonging the debate has been an inability of its proponents to unseat those objections. In fact, as is shown here, all objections can be met and the test as stated is correct. In particular, the role of stationarity as knowledge in addition to the sample values turns out to be crucial. Under certain conditions, including those addressed by the bispectral aliasing test, the continuoustime signals reconstructed from aliased samples are nonstationary. Therefore detecting aliasing in (at least some) stationary continuous-time processes both makes sense and can be done. The merits of the bispectral test for practical use are briefly addressed, but our primary concern here is its theoretical soundness.
\end{abstract}

\section{THE BISPECTRAL ALIASING TEST}

The domain of the discrete-time bispectrum is the two dimensional bifrequency $\left\{\omega_{1}, \omega_{2}\right\}$ plane. Assuming a realvalued discrete time series, the usual replication phenomenon dictates that all non-redundant information is confined to the square $0 \leq \omega_{1}, \omega_{2} \leq \pi$. When one fully accounts for symmetries, the non-redundant information in the bispectrum is confined to a particular triangle inside this square [1, 6].

This triangle naturally divides into two pieces. One piece is an isosceles triangle and is unproblematic. The other piece, somewhat unusual in shape, is the source of the controversy under discussion. Naive consideration of this triangle shows that it involves frequencies higher than the Nyquist

This work was supported by Los Alamos National Laboratory LDRD 97028. frequency and therefore must have something to do with aliasing. Hinich and Wolinsky considered this more carefully and showed that the naive intuition is correct: if the discrete time series arises from sampling a stationary, bandlimited, continuous-time process, and if the sampling rate is sufficiently rapid to avoid aliasing, then the discrete bispectrum is non-zero only in the isosceles triangular subset of the fundamental domain. Conversely, if the bispectrum of a sampled stationary continuous-time process is non-zero in the outer triangle, then the sampling rate was too slow to avoid aliasing.

It should be clearly understood that there is no assertion that aliasing in general can be detected. The statement is not "if a signal is aliased, then the outer triangle will have a non-zero bispectrum." Rather, the assertion is the converse, "if the outer-triangle shows a non-zero bispectrum, the (underlying) continuous-time signal must have been aliased."

At one level, this result is obvious and, in fact, the result was initially so-regarded [6]. However, doubt soon arose. Perhaps the most important source for suspicion is the argument based on reconstruction alluded to above.

In light of this objection, one is led to reconsider the association of the outer triangle with aliasing. One can take the position that there is no relation, as in [2]. One can decide that something is aliased, but that it is the bispectral estimator rather than the signal. There is some plausibility to this claim, for the frequencies that are involved in the outer triangle are $\omega_{1}, \omega_{2}$, and $\omega_{1}+\omega_{2}-2 \pi$. This seems to be the position of Pflug et al. [5].

Or, one can try to delineate the conditions, if any, under which the test makes sense. This was done by Hinich and Messer in 1995[4]. They confirmed the validity of the original argument and stated its conclusions more carefully. In particular they conclude that a non-zero bispectrum in the outer triangle indicates a non-random signal or one of the following:

- a random, but non-stationary signal ;

- a random, stationary, but aliased signal, or;

- a random, stationary, properly-sampled signal which violates the mixing condition. 
We believe that the analysis of Hinich and Messer, while entirely correct, did little to persuade the detractors of the test. In particular their analysis did not address the reconstruction objection and may have left the impression that the circumstances for which the test applies are unlikely to be met in practice.

In this paper, we show that the reconstruction objection is far from fatal. We further establish that stationarity is the only property which is crucial to the test. Since this property is required in order to define the bispectrum, one can legitimately apply the aliasing test whenever one is entitled to compute a bispectrum. Therefore the bispectral aliasing test is as theoretically sound as the bispectrum itself.

\section{THE SELECTION RULE AND BRILLINGER'S FORMULA}

The bispectrum, defined to be the triple Fourier transform of the third-order autocorrelation, reduces to a function of two frequencies since stationarity confines the spectrum to the plane through the origin of the frequency domain perpendicular to the vector $(1,1,1)$.

$$
\mathfrak{F}_{123}\left(c_{3}\left(t_{1}, t_{2}, t_{3}\right)\right)=b\left(\omega_{1}, \omega_{2}\right) \delta\left(\omega_{1}+\omega_{2}+\omega_{3}\right)
$$

Another way of computing the bispectrum is to switch the order in which one does the Fourier transforming and the ensemble averaging. This leads to the following result.

$$
b\left(\omega_{1}, \omega_{2}\right)=\left\langle X\left(\omega_{1}\right) X\left(\omega_{2}\right) X\left(\omega_{3}=\omega_{1}+\omega_{2}\right)\right\rangle
$$

If the process is bandlimited and $X(\omega)=0$ for $|\omega|>\pi$, then the bispectrum is confined to the intersection of the $(1,1,1)$ plane and the $\pi$-cube (i.e. $\left(\omega_{1}, \omega_{2}, \omega_{3}\right) \in(-\pi, \pi) \otimes$ $(-\pi, \pi) \otimes(-\pi, \pi))$. The plane and its projection onto the $\left(\omega_{1}, \omega_{2}\right)$ plane is shown in Figure 1. Upon sampling with unit time step, one obtains the usual replication in three dimensions. (Doing everything in 3-dimensions and projecting at the end keeps things simpler and makes it easier to avoid errors.) In particular, one gets that if the process is sampled at a frequency greater than twice the highest frequency component, then the bispectrum is confined to the replications of the tilted hexagon shown.

The replication gives the discrete-time bispectrum $b_{d}$ :

$$
b_{d}\left(\lambda_{1}, \lambda_{2}, \lambda_{3}\right)=\sum_{\omega_{1}+\omega_{2}+\omega_{3}=0} b\left(\omega_{1}, \omega_{2}, \omega_{3}\right) .
$$

where $\omega_{i}=\lambda_{i}+2 \pi k$ for integer $k$.

Since the replication does not cause any overlaps, the outer triangle remains empty. This is the Hinich and Wolinsky aliasing theorem. (Note that the outer triangle is equivalent to the bigger triangle with vertices $(0, \pi, 0),(0,0, \pi)$ and $(0, \pi, \pi)$ by symmetries. See [1, 9 ] for details.)

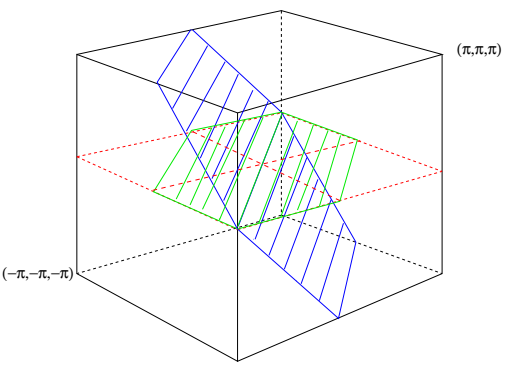

Figure 1: The origin of the bispectral fundamental domain.

\section{THE RECONSTRUCTION OBJECTION}

Suppose we have a stationary process $x(t)$ and we undersample it by sampling at $t \in Z$. Then by convolving $x(t)$ with the appropriate $\operatorname{sinc}$ function we get a reconstructed process $x_{r}(t)$. This new process will have exactly the same samples as the original process and therefore exactly the same sampled bispectrum: yet it is not aliased. Therefore for any process that is undersampled, we have another process producing an identical sampled process which is not undersampled, showing that that one could not possibly detect aliasing via the bispectrum computed from samples!

The rub here is the fact that the reconstructed signal will not necessarily be stationary. Processes reconstructed from aliased samples of continuous-time signals are generally cyclostationary but not stationary. Some aliased processes do, in fact, reconstruct into stationary processes. But in the class of stationary signals for which the bispectral aliasing test gives positive results, reconstruction from aliased samples produces non-stationary processes.

To carefully illustrate this we will consider several stationary processes generated by taking a periodic signal with period $\mathrm{T}$ and giving it a random shift $\theta \in[0, \mathrm{~T})$. First consider a simple cosine process,

$$
x(t)=\cos (\alpha \pi t+2 \pi \theta / T),
$$

where $\alpha=1.5, \mathrm{~T}=4 / 3$, and $\theta$ is randomly chosen from $[0,4 / 3)$. Upon sampling and reconstruction we get the cosine process given by

$$
x_{r}(t)=\cos (\hat{\alpha} \pi t+2 \pi \theta / T),
$$

where $\hat{\alpha}=-0.5$. The key idea is that the signal appears at the lower frequency as dictated by its replication into the fundamental region of the frequency space, $(-\pi, \pi)$, but its reconstructed phase is the same as the "source" component phase.

Now consider

$$
x(t)=\cos (\alpha \pi t+2 \pi \alpha \theta)+\cos (\beta \pi t+2 \pi \beta \theta),
$$


where $\alpha=1.0, \beta=3.0$, and $\theta$ is chosen randomly from the interval $\left[0, \alpha^{-1}\right)$. The reconstructed process one gets is

$$
x(t)=\cos (\alpha \pi t+2 \pi \alpha \theta)+\cos (\hat{\beta} \pi t+2 \pi \beta \theta),
$$

(where $\hat{\alpha}$ and $\hat{\beta}$ are the aliased frequencies). Although the phase terms $2 \pi \alpha \theta$ and $2 \pi \beta \theta$ are still uniformly distributed over $2 \pi$ they now correspond to different time shifts so we no longer have a single shifted waveform. This process can easily be seen to be cyclostationary, but not stationary. See Figure 2 [8]. Computation of the required expectations requires that one can "average over the ensemble." Since this stationary process is not ergodic, one can not get the result from a single realization of the process. It is at this point that some differences in perspective arise. Strictly speaking, in order to compute a bispectrum one must perform the ensemble average. A single realization does not suffice unless the process is ergodic.

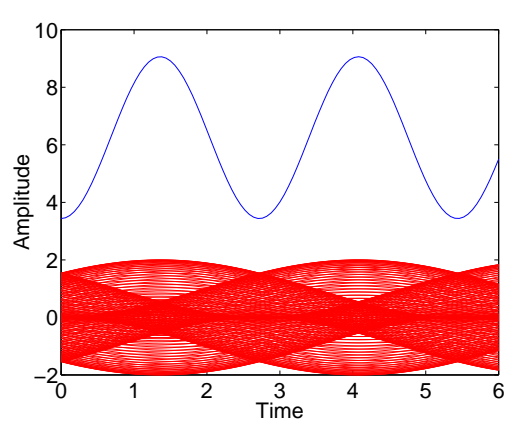

Figure 2: Cyclostationarity of signal reconstructed from aliased samples. The lower curve is the process envelope. The sampling interval is $e$. The upper curve is the sixth moment, chosen for ease of display.

Finally, consider the process defined by

$$
\begin{aligned}
x(t)= & \cos ((10 / 20) \pi t+5 \cdot 2 \pi \theta)+ \\
& \cos ((12 / 20) \pi t+6 \cdot 2 \pi \theta)+ \\
& \cos ((22 / 20) \pi t+11 \cdot 2 \pi \theta)
\end{aligned}
$$

where $\theta$ is chosen randomly from $[0,1)$.

Because the phases of these components are in a fixed relation, this process has a spike in the bispectrum at $\omega_{1}=$ $10 / 20 \pi, \omega_{2}=12 / 20 \pi$, i.e., in the outer triangle. The reconstructed signal is given by

$$
\begin{aligned}
x(t)= & \cos ((10 / 20) \pi t+5 \cdot 2 \pi \theta)+ \\
& \cos ((12 / 20) \pi t+6 \cdot 2 \pi \theta)+ \\
& \cos (-(18 / 20) \pi t+11 \cdot 2 \pi \theta)
\end{aligned}
$$

which is not stationary. Therefore we have a signal with nonempty outer triangle whose reconstruction is not stationary. This situation is exactly what the bispectral test implies happens whenever the outer triangle is nonempty. The loss of stationarity causes the Fourier transform of the triple autocorrelation to "move off" of the $(1,1,1)$ plane.

Therefore, if one knows (or is willing to assume) that the process which generated the observed samples was stationary, one can rule out the unaliased reconstruction as the source of the samples. In a sense, the continuous time signal reconstructed from aliased samples of an original time series is a "measure zero" object. This result is very surprising to most people's intuitions. It is studied further in Vixie, Sigeti and Wolinsky [8].

\section{THE REPLICATION OBJECTION}

Upon looking at Equation 2 one may observe that even if $X(\omega)=0$ for $|\omega|>\pi$, sampling effectively fills in the spectrum at higher frequencies. This is the basis for the objection appearing in Swami [7]. This concern is addressed as follows. While the spectrum does indeed fill out upon sampling, the undesired expectations remain zero. Consider a (statistically stationary) ensemble constructed by uniformly translating a periodic or finite-duration waveform $x(t)$. Two operations are necessary to produce the discrete-time ensemble; a uniform shift in time over a period $T$, which introduces linear phase factors, and sampling, which produces spectrum replication. These operations do not commute: i.e., one wants to time-shift the waveform first and then sample, rather than to shift its samples. For the shifted samples $x_{s}(t+\theta)$ one finds

$$
\mathfrak{F}\left(x_{s}(t+\theta)\right)=e^{i \theta \omega} \mathfrak{F}\left(x_{s}(t)\right)
$$

But for the sampled shifted waveforms $\left.x(t+\theta)\right|_{s}$, i.e., the waveforms needed to construct a stationary ensemble, the phase of the original signal is propagated to higher frequencies periodically rather than linearly. This difference leads to the vanishing of unwanted expectations.

For example, consider the process given by the randomly shifted sum of unit amplitude cosine waves with frequencies at $n / 20(\mathrm{rad} / \mathrm{s})$ where $n$ takes integer values from 1 to 19 . The sampled spectrum has components at $\omega_{1}=10 \pi / 20$, $\omega_{2}=11 \pi / 20$ and $\omega_{3}=-21 \pi / 20$ but the average

$$
\left\langle X\left(\omega_{1}\right) X\left(\omega_{2}\right) X\left(\omega_{3}\right)\right\rangle
$$

reduces to

$$
\left\langle e^{i 10 \theta \pi} e^{i 11 \theta \pi} e^{i 19 \theta \pi}\right\rangle_{\theta}
$$


where $\theta$ is chosen with uniform probability from $[0,1)$. This average vanishes. Therefore, the potential contribution in the outer triangle is zero because averaging kills it. This is in contrast to the case where the average is zero because the spectral amplitudes are themselves zero (as in the proof of the aliasing test).

\section{EMPIRICAL COUNTER-EXAMPLES}

Other objections to the test have been made. Frequently these objections involve a (purported) counter-example to the bispectral aliasing test. A particularly clear example is provided by Frazer, Reilly and Boashash [2]. Here the authors do two things. They present an example of an aliased signal which the aliasing test fails to mark as aliased. The example is unproblematic: neither the aliasing test nor any aliasing test we are aware of will detect all aliased signals. It is not, however, a counter-example to the test. Since there is nothing in the outer triangle, the bispectral aliasing test makes no assertion regarding the presence of aliasing.

The other example the authors provide is more interesting. It consists of a signal involving coupled sinusoids at $\omega_{1}=0.3125 \mathrm{~Hz}, \omega_{2}=0.25 \mathrm{~Hz}$ and $\omega_{3}=.4375 \mathrm{~Hz}$ and the authors show that there is a peak in the outer triangle under conditions which rule out aliasing. As the authors note these frequencies sum to $1 \mathrm{~Hz}$ ((the sampling rate). Under these conditions the authors are correct in asserting that the aliasing test gives a positive result, which they believe to be incorrect. However, what the aliasing test actually indicates is that this signal is non-stationary. The particular interaction which the authors have constructed is not one for which the continuous-time selection criteria is met, i.e., the frequencies involved do not sum to zero. Samples of this signal do meet the discrete-time stationarity condition and so a non-zero bispectrum is possible in the outer triangle.

One can look at these results in various ways. Our position is that neither example constitutes a counter-example to the validity of the aliasing test in theory, though they both show that the test is limited in practice. The first example shows that there are aliased signals which the test does not see. This is obvious anyway since there are signals with zero bispectrum whose samples can be aliased. The second example shows that the term "aliasing test" must be restricted to stationary signals. As stated earlier, this restriction is inherent in the definition of the bispectrum.

\section{CONCLUSIONS}

So, is this something for nothing? How can one get information about higher frequency amplitudes from what is usually thought of as Nyquist-limited data? The answer is of course that the assumption of stationarity is far from nothing. But, to exploit stationarity one must be able to perform the ensemble averaging indicated in the definition of the bispectrum. This implies that one must either have an ergodic process or have access to sufficiently many sample paths.

It is certainly possible that, in practice, the bispectrum can be usefully applied to signals for which there is no theoretical justification. For such uses the aliasing test is silent. However, it is essential that a clear understanding of the fundamental properties of higher-order spectra be available. And the present authors believe that correct understanding of the outer triangle leads to deeper insight of the meaning of the bispectrum in general.

\section{REFERENCES}

[1] David R. Brillinger and Murray Rosenblatt. Computation and interpretation of $\mathrm{k}$-th order spectra. In Bernard Harris, editor, Advanced Seminar on Spectral Analysis of Time Series, volume XV. John Wiley and Sons, Inc., 1967.

[2] G. Frazer, A. Reilly, and B. Boashash. The bispectral aliasing test. Proc. IEEE Workshop Higher-Order Statistics, Lake Tahoe, CA, pages 332-335, June 1993.

[3] M. J. Hinich and M. A. Wolinsky. A test for aliasing using bispectral analysis. J. Amer. Stat. Assoc, 83:499502, June 1988.

[4] Melvin J. Hinich and Hagit Messer. On the principal domain of the discrete bispectrum of a stationary signal. IEEE Trans. Sig. Proc., 43(9):2130-2134, September 1995.

[5] L. A. Pflug, G. E. Ioup, J. W. Ioup, and R. L. Field. Properties of higher-order correlations and spectra for bandlimited, deterministic transients. J. Acoustic Soc. Am., 91(2):975-988, Feb. 1992.

[6] I. Scharfer and H. Messer. The bispectrum of sampled data: Part 1 - detection of the sampling jitter. IEEE Trans. Sig. Proc., 41:296-312, Jan. 1993.

[7] A. Swami. Pitfalls in polyspectra. In Proceedings of the IEEE International Conference on Acoustics, Speech and Signal Processing, volume IV, pages 97-100, 1993.

[8] Kevin R. Vixie, David E. Sigeti, and Murray Wolinsky. Detection of aliasing in persistent signals. Technical report, Los Alamos National Laboratory LA-UR99-2126, May 1999.

[9] M. Wolinsky. Invitation to the bispectrum. Technical report, University of Texas:Applied Research Laboratories: ARL-TR-88-7, February 1988. 